\title{
Cambios mandibulares después de una expansión maxilar rápida. Revisión de la literatura
}

\section{Mandibular changes after rapid maxillary expansion. Literature review}

\section{Resumen}

El objetivo de esta revisión de literatura es reportar los cambios dentoalveolares y esqueléticos del arco mandibular después de una expansión maxilar rápida (EMR) en denticiones mixtas. Se realizó una búsqueda electrónica en las siguientes bases de datos: Medline/ PubMed, Scopus, LILACS y búsqueda manual en las principales revistas de ortodoncia (American Journal of Orthodontics and Dentofacial Orthopedics, The Angle Orthodontics, Seminars in Orthodontics) y en Google Scholar. Los criterios de elegibilidad incluyeron todos los estudios relacionados al tema de la revisión, en inglés y español, sin restricción del año de publicación. De un total de 62 artículos encontrados, se eligieron 10 para ser analizados. Diversos autores coinciden en la presencia de cambios esqueléticos y dentoalveolares espontáneos clínicamente significativos en el arco dental mandibular a corto y largo plazo después de la EMR. Durante el período posterior a la EMR, se pudo observar un desplazamiento anterior mandibular debido a la sobreexpansión del maxilar, además, se registraron cambios dentoalveolares y aumento en el ancho de las arcadas dentarias.

Palabras clave: Expansión maxilar rápida; Maloclusión; Avance mandibular; Técnica de expansión palatina; Anomalías maxilomandibulares (fuente DeCS BIREME).

\begin{abstract}
The objective of this literature review is to report dentoalveolar and skeletal changes of the mandibular arch after rapid maxillary expansion (RMS) in mixed dentitions. An electronic search was carried out in the following databases: Medline/PubMed, Scopus, LILACS as well as manual search in the main orthodontic journals (American Journal of Orthodontics and Dentofacial Orthopedics, The Angle Orthodontics, Seminars in Orthodontics) and Google Scholar. The eligibility criteria included all studies related to the topic being reviewed, in English and Spanish, without restrictions on the year of publication. Of a total of 62 articles found, 10 were chosen to be analyzed. Quite a few authors agree on the presence of clinically significant spontaneous skeletal and dentoalveolar changes, in the mandibular dental arch in short and long term after RMS. During the aftermath of the RMS, an anterior mandibular displacement could be observed due to the overexpansion of the maxilla, in addition, dentoalveolar changes and an increase in the width of the dental arches were recorded.
\end{abstract}

Keywords: Maxillary expansion; Malocclusion; Mandibular advancement; Palatal expansion technique; Jaw abnormalities (source MeSH NLM).

\section{Artículo de Revisión}

Anthuaneth Aliaga-Meza ${ }^{1, a}$, Jessica Arieta-Miranda 1,b

${ }^{1}$ Universidad Nacional Mayor de San Marcos, Facultad de Odontología, Lima, Perú.

a Estudiante de pregrado.

${ }^{\mathrm{b}}$ Especialista en Ortodoncia y Ortopedia Maxilar.

Correspondencia:

Anthuaneth Aliaga-Meza: anthuaneth.aliaga@unmsm. edu.pe

Calle José Félix Bogado 2560. Lima. Perú

ORCID: 0000-0001-6719-7037

\section{Coautora:}

Jessica Arieta-Miranda: jarietam@unmsm.edu.pe ORCID: 0000-0003-3482-8992

\section{Editora:}

Rosse Mary Falcón-Antenucci

Universidad Inca Garcilaso de la Vega, Perú

Conflicto de intereses: los autores declaran no tener conflictos de interés.

Fuente de financiamiento: autofinanciado.

Recibido: $25 / 11 / 20$

Aceptado: $13 / 06 / 21$

Publicado: 05/10/21 


\section{Introducción}

La expansión maxilar rápida (EMR) fue una propuesta introducida por Angell en el siglo XIX ${ }^{1}$. Esta es una técnica muy empleada en el tratamiento de discrepancias entre los arcos maxilares, la cual aumenta el perímetro del arco por medio de la separación de la sutura media palatina, provocando la expansión, que se lleva a cabo cuando la fuerza aplicada origina el ensanchamiento y la apertura gradual de dicha sutura ${ }^{2}$.

Las deficiencias trasversales del arco maxilar están relacionadas comúnmente con la falta de espacio en el arco durante la dentición mixta. Las maloclusiones ocasionadas por estas deficiencias no se autocorrigen durante el crecimiento craneofacial; el diagnóstico y el tratamiento con EMR de esta patología deben realizarse tan pronto como sea posible. Con la finalidad de aumentar transversalmente la medida del arco maxilar, realizar un tratamiento eficaz a edad temprana y evitar el desarrollo de una asimetría facial esquelética resultante de las mordidas cruzadas, que a su vez, pueden conducir a trastornos funcionales y estructurales del sistema estomatognático durante el crecimiento ${ }^{3,4}$.

La constricción espontánea del arco mandibular puede ocurrir como un proceso de adaptación a la constricción maxilar progresiva, observada en pacientes con discrepancias transversales no tratados 5 . Por tanto, se formula la hipótesis de que, si la constricción maxilar induce a la constricción del arco mandibular, con el paso del tiempo la expansión maxilar podría inducir efectos dentoalveolares y esqueléticos espontáneos en el arco mandibular, por ejemplo: el crecimiento de la mandíbula puede incrementarse de 2 a $4 \mathrm{~mm}^{5}$. Esta teoría está basada en que el ensanchamiento del maxilar con una EMR, a menudo, conduce a la postura espontánea hacia adelante de la mandíbula durante el período de retención ${ }^{5,6}$.

La eficacia de la EMR en los parámetros dentales o esqueléticos transversales sigue siendo controversial, y se ha escrito poco sobre los cambios mandibulares posteriores a este procedimiento. Debido a lo expuesto anteriormente, el objetivo de esta revisión de la literatura es investigar la respuesta espontánea del arco mandibular después a la EMR como intervención ortodóntica para el tratamiento de las discrepancias trasversales maxilomandibulares y a su vez, reporter los cambios dentoalveolares y esqueléticos mandibulares espontáneos a corto y largo plazo después de la EMR.

\section{Métodos}

La búsqueda bibliográfica electrónica se realizó en las siguientes bases de datos: Medline/PubMed, Scopus, LILACS y búsqueda manual en las principales revistas de ortodoncia (American Journal of Orthodontics and Dentofacial Orthopedics (AJO-DO), The Angle Orthodontics, Seminars in Orthodontics) y en Google Scholar, usando las palabras clave: "expansión maxilar rápida", "maloclusión", "avance mandibular", "técnica de expansión palatina", "anomalías maxilomandibulares" y sus homólogos en inglés. La búsqueda se complementó con las referencias de los artículos incluidos. Se recuperaron un total de 62 artículos, se suprimieron los documentos duplicados y fue realizada la selección de los artículos por medio de los criterios de elegibilidad. Los criterios de elegibilidad incluyeron todos los estudios relacionados al tema específico de la revisión, escritos en inglés y español, sin restricción del año de publicación. Se verificó la relevancia de los títulos y resúmenes de los artículos obtenidos para luego descargar el texto completo. De esta manera fueron seleccionados 10 artículos para la revisión de literatura (Tabla).

\section{Revisión de la literatura}

Discrepancia transversal maxilar. La discrepancia transversal del maxilar constituye uno de los problemas esqueléticos más generalizados en la región craneofacial 5. Por lo general, un arco maxilar con un ancho transpalatino de 36 a $39 \mathrm{~mm}$, permite acomodar una dentición de tamaño promedio sin presentarse apiñamiento o diastemas, mientras que los arcos maxilares de menos de $31 \mathrm{~mm}$ de ancho pueden causar apiñamiento, por lo tanto, necesitarán una expansión ortopédica o asistida quirúrgicamente. Adicionalmente a la mordida cruzada, el apińamiento constituye uno de los problemas más comunes que se encuentran como consecuencia de la discrepancia en los arcos dentales (entre el tamaño de los dientes y las bases óseas) ${ }^{5}$. Si en la dentición maxilar se presenta una discrepancia transversal, es muy probable que se produzca mordida cruzada; pero, si la constricción maxilar está camuflada por la dentición y ambas arcadas dentarias se constriñen al mismo tiempo, se observa apińamiento en ausencia de mordida cruzada ${ }^{5}$. Por lo tanto, es de vital importancia, evaluar el esqueleto craneofacial en la dimensión transversal lo más temprano posible y diagnosticar con precisión la necesidad de expansión transversal, para mejorar la eficiencia y efectividad del tratamiento.

El diagnóstico de estas alteraciones trasversales no es sencillo, y a menudo incluye más de un método: observación clínica detallada, análisis de yeso dental y exámenes complementarios, como telerradiografía posteroanterior y tomografía computarizada de haz cónico (CBCT), considerada el examen auxiliar de elección para el diagnóstico de discrepancia transversal del maxilar ${ }^{7}$.-

Diagnóstico diferencial entre una discrepancia transversal esquelética vs dentaria. Existen diferentes maneras de diagnosticar la dimensión transversal de las bases óseas, a nivel de los procesos alveolares, uno de los exámenes clásicos fue el descrito por Schwarz en 1966, basado en el análisis de las inclinaciones axiales vestíbulolinguales de los dientes posteriores. Este método visual, orienta sobre la existencia de un problema transversal y permite valorar si el problema es dentario, esquelético o mixto ${ }^{8}$.

No obstante, Ricketts ${ }^{9}$ (1981), enfatizó el uso del análisis frontal para comparar y determinar la diferencia del ancho entre las bases óseas. Así, propuso medir la severidad de la discrepancia transversal y distinguir si el problema es de origen dental o esquelético, mediante el uso de la telerradiografía frontal de cráneo. El análisis 


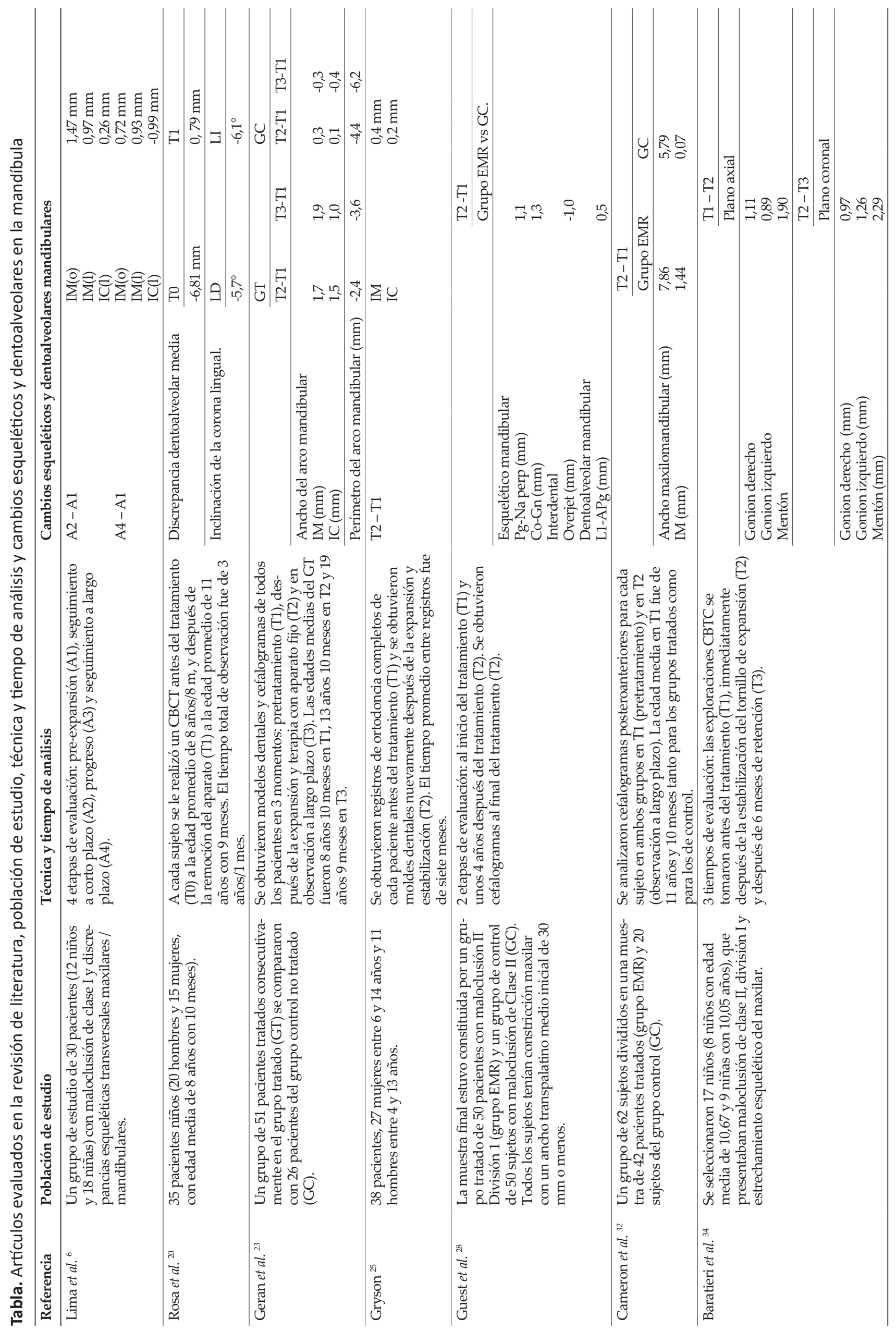




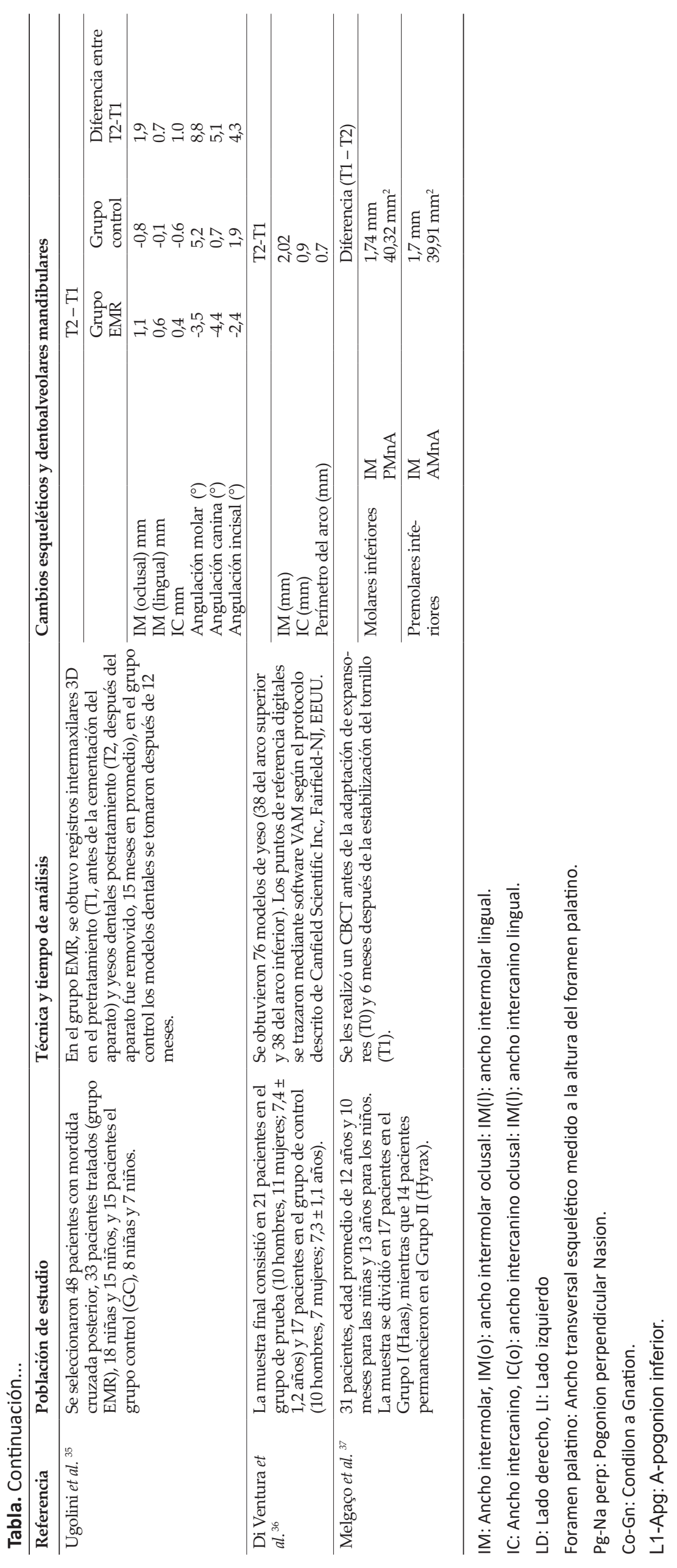


del problema esquelético transversal maxilomandibular, según este autor, se realiza mediante la medición de la distancia entre el punto JR al plano facial frontal derecho $(\mathrm{GA}-\mathrm{ZR})$ y JL al plano facial frontal izquierdo (AG - ZL). Además, reportó que el problema transversal debe ser analizado de forma independiente en cada lado (derecho e izquierdo) y viceversa ${ }^{10}$. En cada lado la distancia ha de ser la misma $10 \mathrm{~mm}+/-1,5 \mathrm{~mm}$. El ancho mandibular (AG-GA) en adultos tiene una media de $88,5 \mathrm{~mm}+/-3 \mathrm{~mm}$ y el ancho maxilar, de $70 \mathrm{~mm}+/-3$ $\mathrm{mm}$, la diferencia obtenida entre ambos valores es la misma diferencia entre las bases óseas, este valor en el adulto sería $18,5 \mathrm{~mm}+/-3 \mathrm{~mm}^{9,10}$.

McNamara ${ }^{5}$, analizó la dimensión transversal mediante el ancho intermolar, afirmando que es un indicativo del desarrollo de la base ósea maxilar. Los resultados mostraron que en la dentición mixta, el ancho era de 34-35 $\mathrm{mm}$ y 36-39 $\mathrm{mm}$ en adultos con dentición permanente. Howe y McNamara ${ }^{11}$ y McNamara ${ }^{12}$ al observar que las arcadas sin apiñamiento eran notablemente más amplias que las arcadas con apiñamiento; demostraron que el ancho y espacio disponible en una arcada correctamente desarrollada transversalmente debe permitir alojar y acomodar adecuadamente a la dentición permanente.

Los cefalogramas postero-anteriores son considerados la forma más fácil y confiable de evaluar las discrepancias esqueléticas transversales ${ }^{13}$. Sin embargo, estas imágenes bidimensionales (2D) tienen limitaciones técnicas que afectan la precisión al momento de ubicar los puntos de referencia, generando importantes errores de identificación de los puntos craneométricos ${ }^{14}$.

El surgimiento de imágenes tridimensionales (3D), como una modalidad de diagnóstico efectiva de las deficiencias transversales maxilares pueden ser muy útiles. Porque no son afectadas por las limitaciones técnicas asociadas con el cefalograma postero-anterior 2D, el uso de esta tecnología ha demostrado una variabilidad menor y más reproducibilidad de las mediciones transversales en cefalogramas posteroanteriores construidas a partir de CBCT, en comparación con el cefalograma postero-anterior 2D convencional. La capacidad de realizar cortes radiográficos transversales localizados y específicos de imágenes $\mathrm{CBCT}$, para evaluar áreas de interés, también tiene un potencial significativo en el diagnóstico de la dimensión transversal craneofacial ${ }^{15}$.

Una vez diagnosticado un arco maxilar con constricción esquelética, el tratamiento de elección es la expansión esquelética ortopédica, que involucra separación de la sutura media palatina ${ }^{16}$. Hasta hace poco existían tres alternativas de tratamiento disponibles para este propósito: EMR, expansión maxilar lenta (EML) y EMR asistida por cirugía (SARPE) ${ }^{16}$. Tanto EML como EMR están indicados para pacientes en crecimiento, mientras que SARPE es una alternativa en el tratamiento de pacientes adolescentes y adultos jóvenes que cesaron su crecimiento ${ }^{16}$.

Recientemente con el desarrollo de técnicas digitales, se ha demostrado que es posible la expansión del maxilar en pacientes adultos sin realizar osteotomías, utilizando anclaje mediante minitornillos ${ }^{17,18}$. Esta EMR asistida por minitornillos (MARPE), es un enfoque novedoso y menos invasivo que cumple con el objetivo de separar la sutura media palatina sin causar efectos colaterales a nivel de los dientes y el periodonto ${ }^{18}$. Al respecto, Lee $e t$ al. ${ }^{18}$ concluyen que esta nueva técnica se configura como una modalidad de tratamiento eficaz utilizada para la corrección transversal y que podría eliminar la necesidad de procedimientos quirúrgicos invasivos, aprovechando así las posibilidades que ofrecen las suturas.

La EMR es eficaz para corregir problemas de mordida cruzada transversal y proporcionar suficiente espacio en el arco dentario para resolver el apiñamiento en pacientes de dentición mixta. Además, este procedimiento se puede utilizar para aplanar la curva de Wilson, mejorar la función de las vías respiratorias nasales y corregir el corredor bucal con fines estéticos 5 .

EMR como tratamiento de las discrepancias maxilares en dentición mixta. Estudios han confirmado que el tratamiento con EMR, durante la dentición mixta, es efectiva en mordidas cruzadas y discrepancias maxilares, además, se determinó que el aumento de la longitud del arco, puede mejorar el alineamiento de los dientes superiores, corrigiendo el apińamiento ${ }^{19,20}$.

Rosa et al. ${ }^{20}$, por medio de un estudio clínico prospectivo, evalúo 35 pacientes que presentaban apiñamiento de los incisivos superiores permanentes en dentición mixta temprana con ausencia de mordida cruzada posterior. Fue aplicado el protocolo de EMR en los pacientes y se logró la corrección del apiñamiento de incisivos en todos los casos, siendo la discrepancia dentoalveolar media de $-6,81 \mathrm{~mm}$ antes del tratamiento (T0) y $0,79 \mathrm{~mm}$ después del retiro del aparato (T1). Hubo un aumento del ancho transversal esquelético medido a la altura del foramen palatino de $2,5 \mathrm{~mm}$. La mordida cruzada anterior se autocorrigió en todos los pacientes durante la expansión activa; los primeros molares permanentes inferiores se enderezaron en dirección vestibular y mostraron una disminución promedio de la inclinación de la corona lingual de $-5,7^{\circ}$ para el lado derecho y de $-6,1^{\circ}$ para el izquierdo.

Desde la década de los 90's, algunos estudios ${ }^{21,22}$ intentaron describir los efectos del tratamiento y la estabilidad a corto y largo plazo de la EMR utilizada en la dentición mixta temprana. En 1995, Spillane y McNamara ${ }^{21}$ analizaron modelos dentales de 162 pacientes para medir las dimensiones del arco antes e inmediatamente después de la expansión y a intervalos anuales hasta la erupción de los primeros premolares. Observaron un aumento considerable de 5 a $6 \mathrm{~mm}$ del ancho transpalatal. Durante el periodo posterior a la expansión, la mayoría de los aumentos en el ancho del arco se mantuvieron estables, el 90,5\% de la expansión original en los primeros molares permanentes se mantuvo después del primer año, y el 80,4\% después de 2,4 años de la expansión. Del mismo modo, en 1999 Memikoglu y I-eri ${ }^{22}$ reportaron en su estudio que los anchos de las bases maxilares aumentaron significativamente en $4,57 \mathrm{~mm}$ 
al final del tratamiento, y el ancho intermolar superior aumentó en 5,02 mm durante el tratamiento con EMR. Los anchos intermolares e intercaninos superiores medidos a partir de los modelos dentales, también aumentaron en 3,42 mm y 5,42 mm, respectivamente, al final del tratamiento.

Geran et al. ${ }^{23}$ evaluaron la estabilidad a largo plazo de los cambios en las arcadas dentarias inducidas por el EMR en la dentición mixta temprana, seguido de un tratamiento integral de ortodoncia con aparatología fija. La evaluación final de los pacientes se realizó 10 años después de la EMR. Se compararon modelos dentales en serie de los arcos dentarios con el grupo control no tratado. En los resultados se observó aproximadamente $4 \mathrm{~mm}$ de aumento en el perímetro del arco maxilar y 2,5 $\mathrm{mm}$ en el arco mandibular. Además, a largo plazo hubo un incremento en el ancho intermolar de $1,9 \mathrm{~mm}$ y en el ancho intercanino de $1 \mathrm{~mm}$, en comparación con el grupo control donde al contrario se observó una disminución de -0,3 mm y -0,4 mm en el ancho intermolar e intercanino, respectivamente.

Según los resultados obtenidos de estos estudios ${ }^{21-23}$, la terapia con EMR en la dentición mixta, es un enfoque de tratamiento eficaz para corregir las deficiencias transversales en el arco maxilar y mandibular a largo plazo, a pesar de que la EMR no se aplicó directamente en la mandíbula. De estudios como éstos, nace la interrogante de parte de los autores para poder investigar más a fondo los efectos dentoalveolares y esqueléticos que puede producir la EMR en otras estructuras además de la maxila, especialmente en la mandíbula.

Efectos dentoalveolares y esqueléticos de la EMR sobre la mandíbula. Durante mucho tiempo se investigó las discrepancias transversales en los arcos dentarios, sus posibles tratamientos y efectos. Los efectos no solo están presentes en el hueso maxilar, sino también en el ancho de la cavidad nasal, en los procesos maxilares propiamente dichos, además se observó efectos esqueléticos y dentoalveolares mandibulares, como la tendencia de la mandíbula a desplazarse hacia abajo y atrás y el aumento del ancho intermolar mandibular en $0,4 \mathrm{~mm}$ y el ancho intercanino mandibular en $0,2 \mathrm{~mm}^{24,25}$.

Tollaro et al. ${ }^{26}$ encontraron una discrepancia transversal posterior de 3 a $5 \mathrm{~mm}$ en sujetos con dentición mixta temprana y maloclusiones clase II sin mordidas cruzadas posteriores en relación céntrica (RC). Esta discrepancia transversal se podía observar clínicamente al protruir; debido a esto se postuló que la mandíbula se mantiene en una posición distal, porque el maxilar constreñido lo está reteniendo, impidiéndole, al mismo tiempo, asumir la posición de $\mathrm{RC}^{27}$. La presencia de una discrepancia transversal primitiva entre los arcos dentales induce una posición hacia atrás de la mandíbula, para así obtener el mayor número de contactos interdentarios ${ }^{5}$.

Otros estudios ${ }^{28,29}$ determinaron que el ensanchamiento del maxilar con EMR induce a una postura espontánea hacia adelante de la mandíbula, durante el período de retención. La expansión elimina las interferencias oclusales, permitiendo que la mandíbula se proyecte hacia adelante y aumente su longitud, mejorando así las relaciones intermaxilares. Reichenbach y Taatz ${ }^{30}$ utilizaron el ejemplo de un pie y el calzado, donde el pie representa a la mandíbula y el calzado al maxilar. Cuando el calzado es demasiado angosto es imposible que el pie asiente por completo, después de que el "calzado" se ensancha, la mandíbula que actúa como el "pie" se mueve hacia adelante en su posición correcta.

Una explicación alternativa para los cambios dentoalveolares en la mandíbula después de la terapia con EMR, es que acompaña los cambios en la orientación de los planos inclinados de los dientes. Haas ${ }^{31}$ en 1961, postuló que estos cambios en el arco mandibular son el resultado de alteraciones en el equilibrio entre la lengua y los músculos buccinadores.

Años más tarde, Lima et al. ${ }^{6}$ analisaron la respuesta mandibular espontánea a corto y largo plazo después de la EMR en pacientes con maloclusiones de clase I y discrepancias esqueléticas transversales maxilares/mandibulares, determinando que, desde la pre-expansión hasta el seguimiento a corto plazo, se observó un aumento de $1,47 \mathrm{~mm}$ en el ancho intermolar mandibular oclusal, $0,97 \mathrm{~mm}$ en el ancho intermolar lingual y $0,26 \mathrm{~mm}$ en el ancho intercanino lingual. Para determinar la asociación entre los cambios y el crecimiento normal, las medidas de ancho oclusal de cada paciente se restaron de los cambios de ancho medio de Moorrees bilateralmente y para la edad y el sexo de cada niño. Obteniendo aumento de $0,08 \mathrm{~mm}$ en el ancho intermolar mandibular (oclusal), que podría atribuirse a cambios a través del crecimiento normal y $1,39 \mathrm{~mm}$ (de 1,47 mm) a la EMR. En el seguimiento a largo plazo, el ancho intermolar mandibular tuvo un aumento significativo en $0,72 \mathrm{~mm}$ y $0,93 \mathrm{~mm}$ en oclusal y lingual, respectivamente, en comparación con la pre-expansión, lo que indica que el aumento de la dimensión del ancho del arco mandibular fue en respuesta a los efectos ortopédicos de la EMR, incluso en la transición de la dentición mixta a la permanente, es decir, permanecieron estables durante todas las observaciones en distintos periodos de tratamiento.

De igual manera, Cameron et al. ${ }^{32}$ reportaron que la EMR indujo cambios relevantes tanto en las estructuras maxilares como en otras regiones craneofaciales. Hubo incremento del ancho maxilomandibular de 7,86 $\mathrm{mm}$ en el grupo tratado, en comparación con el grupo control donde se observó un incremento de $5,79 \mathrm{~mm}$. Además, hubo incremento de $1,4 \mathrm{~mm}$ a largo plazo en el ancho intermolar mandibular. Esto reveló una sobrecorrección de la deficiencia inicial en el ancho intermolar mandibular de $0,8 \mathrm{~mm}$.

Lione et al. ${ }^{33}$ no encontraron diferencias estadísticamente significativas para la posición sagital del maxilar y la mandíbula al final del tratamiento con EMR. Los pacientes tratados con EMR, no mostraron ninguna mejoría destacable de la relación anteroposterior del maxilar y de la mandíbula a niveles esqueléticos y oclusales, en comparación con el grupo control. 
A pesar de la variabilidad de los resultados que los autores indican, las investigaciones ${ }^{6,32,33}$ han demostrado la existencia de una posible relación entre la EMR y cambios dentoalveolares y del ancho del arco mandibular. Sin embargo, aún no se ha escrito lo suficiente sobre el comportamiento de la mandíbula después de la terapia con EMR $5,6,25,28-30,32,33$.

\section{Evaluación tridimensional de los efectos de la EMR} en la mandíbula. La gran mayoría de los estudios anteriormente citados basaron su evaluación en las medidas obtenidas de modelos dentales de yeso convencionales. Por tanto, es necesario evaluar los cambios post EMR, con la utilización de CBCT. Esta técnica permite obtener alta resolución en un período muy corto y con mínima exposición a la radiación, además permite obtener imágenes multiplanares 7,15 .

McNamara ${ }^{5}$ reportó que la posición de la arcada mandibular podría verse influenciada más por la morfología esquelética maxilar que por el tamaño y la forma de la mandíbula. Esta afirmación, explicaría porque ocurre descompensación del arco mandibular durante la terapia de EMR, aunque este tratamiento ha sido ampliamente discutido en la literatura, para la correcta evaluación de la afectación del arco mandibular durante la EMR, algunos autores se preocuparon en realizar una evaluación clara basada en análisis tridimensionales ${ }^{34-36}$.

Baratieri et al. ${ }^{34}$ realizaron un estudio utilizando la CBCT para evaluar el posicionamiento mandibular espacial después de la EMR en la maloclusión Clase II División 1. Se evaluó a 17 niños que presentaban constricción esquelética del maxilar y se sometieron al protocolo de EMR. La evaluación mandibular 3D inmediatamente después de la EMR, mostró en relación con el plano coronal, que gonion izquierdo y derecho avanzaron $0,97 \mathrm{~mm}$ y mentón tuvo un mayor desplazamiento hacia adelante de $2,29 \mathrm{~mm}$, es decir, un posicionamiento significativamente hacia adelante de la mandíbula, exhibiendo una posición más anterior en comparación con la condición inicial. De la misma manera, Ugolini et al. ${ }^{35}$, evaluaron pacientes con mordida cruzada posterior unilateral, mediante modelos digitales 3D y determinaron que la terapia con EMR permitió un incremento en las dimensiones transversales del arco mandibular y en las angulaciones molares, caninas e incisivas. La distancia intermolar mandibular aumentó sustancialmente $1,9 \mathrm{~mm}$ en el lado vestibular y $0,7 \mathrm{~mm}$ en el lado lingual y la angulación de los molares mandibulares aumentó $9^{\circ}$.

Di Ventura et al. ${ }^{36}$ evaluaron tridimensionalmente los efectos de la EMR anclada a molares primarios como una alternativa al tratamiento convencional a la EMR anclada a dientes permanentes. Así, el ancho intermolar aumentó significativamente $2,02 \mathrm{~mm}$ en la mandíbula, $1,39 \mathrm{~mm}$ y $1,75 \mathrm{~mm}$ en los primeros y segundos molares temporales, respectivamente. El ancho intercanino tuvo un aumento de $0,9 \mathrm{~mm}$ y la longitud total del arco de $0,7 \mathrm{~mm}$. Verificando que, la EMR anclada a molares primarios, es una opción de tratamiento eficaz para corregir las deficiencias transversales maxilares y mandibulares.
Melgaço et al. ${ }^{37}$ evaluó tridimensionalmente a 31 pacientes con edad promedio de 12 años y 10 meses (niñas) y 13 años (niños). La muestra se dividió en 17 pacientes en el Grupo I (Haas), mientras que 14 pacientes permanecieron en el Grupo II (Hyrax). Las distancias intermolares e interpremolares de la mandíbula aumentaron $1,74 \mathrm{~mm}$ y $1,7 \mathrm{~mm}$, respectivamente. Todas las áreas mandibulares aumentaron notablemente, el área seccional posterior (PMnA) y anterior de la mandíbula (AMnA) aumentaron 40,32 $\mathrm{mm}^{2}$ y 39,91 $\mathrm{mm}^{2}$, respectivamente. El área oclusal mandibular (MnOA) aumentó en $43,99 \mathrm{~mm}^{2}$ y el contorno oclusal mandibular (MnOC) aumentó en 1,35 mm. En promedio, los incisivos mandibulares disminuyeron su inclinación en $1,23^{\circ}$. Los expansores Haas y Hyrax arrojaron resultados similares, suponiendo así que los efectos de la EMR parecen ser similares independientemente del dispositivo.

\section{Alternativas de tratamiento a la terapia de EMR para las discrepancias transversales maxilomandibulares.} Una alternativa de tratamiento a pacientes que necesiten expansión de ambos arcos por discrepancias transversales y/o apiñamiento es el EMR combinado con el aparato de Schwartz (EMR-Sz) ${ }^{38}$. Antes de iniciar la terapia de EMR es recomendable haber completado, o bien estar por terminar, la terapia con el aparato de Schwarz, con el objeto de que los dientes inferiores estén en posición adecuada al iniciar la terapia de expansión. Se reportó que, al iniciar la primera fase con la descompensación dental mandibular, es posible obtener una mayor expansión transversal del arco dentario superior, disminuyendo la necesidad de una fase adicional en arcos severamente estrechos ${ }^{38}$

O'Grady et al. ${ }^{39}$ evaluaron los cambios a corto y largo plazo en las dimensiones de las arcadas dentarias en pacientes tratados solo con EMR o con EMR-Sz en la dentición mixta temprana, con una edad promedio de 9 años al inicio del tratamiento, seguida por aparatos fijos en la dentición permanente. Se compararon pacientes con EMR (27) y EMR-Sz (23), se observó que el EMR-Sz condujo a resultados considerablemente más favorables que la EMR, hubo mayor aumento del ancho transversal y el perímetro del arco mandibular a largo plazo, enderezado de los dientes posteriores mandibulares, lo que permitió la expansión maxilar clínicamente efectiva para la corrección de discrepancias moderadas arco/diente. Años más tarde, estudios mediante análisis de $\mathrm{CBCT}$ reportaron que el aparato de Schwartz afecta principalmente al complejo dentoalveolar (inclinaciones dentarias, anchos interdentales), y no hubo afectación esquelética considerable en el ancho del cuerpo mandibular ${ }^{40,41}$.

Estas investigaciones ${ }^{39-41}$ confirman la eficacia de la EMR-Sz sobre los componentes dentoalveolares del arco mandibular. Los aumentos relativamente modestos en la dimensión del arco indican que la EMR-Sz puede ser útil en aquellos pacientes con apińamiento leve o moderado de los incisivos inferiores, pero sin satisfacer los requerimientos de longitud del arco en pacientes con apińamiento severo ${ }^{12,38}$. 
Los efectos dentoalveolares provocados por las deficiencias transversales mandibulares en pacientes en crecimiento, habitualmente, se corrigen con la expansión usando dispositivos de Schwarz, estas terapias muestran resultados relativamente estables para pacientes más jóvenes ${ }^{39-41}$. Para pacientes adultos que han cesado su crecimiento y que buscan lograr buenos efectos a nivel esquelético, se ha propuesto la distracción osteogénica de la sínfisis mandibular (DOSM) ${ }^{42}$. Autores pioneros ${ }_{43,44}$ en el uso de la DOSM, demostraron que el procedimiento mandibular que utiliza dispositivos de expansión tanto dentales como óseos, proporciona una alternativa eficaz a la cirugía ortognática.

Del Santo et al. ${ }^{44}$ observó que la osteogénesis por distracción sin un tratamiento previo de ortodoncia produjo inclinación mesial de los incisivos mandibulares, aunque mínima, esta puede poner en riesgo el resultado obtenido. Además, las raíces deben divergir para crear un espacio adecuado para el corte óseo o se debe elegir un lugar diferente. Los brackets deben ser colocados sobre los dientes con la cara mesial en una posición más alta que la cara distal. De esta manera el arco inclina los ápices de la raíz distalmente, abriendo espacio entre ellos.

La DOSM es una modalidad de tratamiento segura y eficaz que debe ser específico para cada caso, así mismo es un procedimiento menos invasivo y logra el objetivo óptimo de tratamiento en el menor período de tiempo con menos riesgo de complicaciones biológicas y técnicas ${ }^{45}$.

\section{Conclusiones}

De acuerdo con el análisis realizado, se puede concluir que: la mayoría de los estudios analizados mostraron que hubo cambios esqueléticos significativos en las bases óseas en pacientes en crecimiento sometidos a EMR, como el desplazamiento anterior de la mandíbula, incremento en las dimensiones transversales (ancho intermolar e intercanino) del arco mandibular y el aumento del perímetro de las arcadas dentarias, mejorando las condiciones de apińamiento y mordida cruzada. Además, después de una terapia con EMR, ocurrieron cambios dentoalveolares en el arco mandibular a corto y largo plazo, demostrando su eficacia en el tratamiento de las discrepancias transversales maxilomandibulares.

\section{Referencias bibliográficas}

1. Angell EH. Treatment of irregularities of the permanent or adult teeth. Dental Cosmos [Internet]. 1860 [citado 22 de agosto del 2020]; 1(9):540-600. Disponible en: http://www.fo.usp.br/sdo/wp-content/uploads/Ange11-pt1.pdf.

2. Al-Battikki R. Rapid maxillary expansion: review of literature. Saudi DentJ [Internet]. 2001 [citado el 23 de agosto del 2020]; 13:1617. Disponible en: https:// scholar.google.com/scholar_lookup?journal=+Saudi+Dent+J\&title=Rapid+maxillary+expansion:+Review+o$\mathrm{f}+$ literature\&author $=\mathrm{R}+\mathrm{Al}+\mathrm{Battikki \& volume}=+13 \& \mathrm{pu}-$ blication_year $=2001 \&$ pages $=+161 \% \mathrm{E} 2 \% 80 \% 93167 \&$.
3. Sandikçioğlu M, Hazar S. Skeletal and dental changes after maxillary expansion in the mixed dentition. Am J Orthod Dentofacial Orthop. 1997;111(3):321-7. DOI: 10.1016/s0889-5406(97)70191-4.

4. McNamara JA Jr, Lione R, Franchi L, Angelieri F, Cevidanes LHS, Darendeliler MA, et al. The role of rapid maxillary expansion in the promotion of oral and general health. Prog Orthod. 2015;16:33. DOI: 10.1186/ s40510-015-0105-x.

5. McNamara JA. Maxillary transverse deficiency. Am J Orthod Dentofacial Orthop. 2000;117(5):567-70. DOI: 10.1016/s0889-5406(00)70202-2.

6. Lima AC, Lima AL, Filho RM, Oyen OJ. Spontaneous mandibular arch response after rapid palatal expansion: a long-term study on Class I malocclusion. Am J Orthod Dentofacial Orthop. 2004;126(5):576-82. DOI: 10.1016/j.ajodo.2004.06.011.

7. Chung $\mathrm{CH}$. Diagnosis of transverse problems. Seminars in Orthodontics. 2019;25(1):16-23. DOI: 10.1053/j. sodo.2019.02.003.

8. Schwarz M, Gratzinger M. Removable Orthodontic Appliances. Philadelphia: W.B. Saunders; 1966.

9. Ricketts RM. Perspectives in the clinical application of cephalometrics. The first fifty years. Angle Orthod. 1981;51(2):115-50. DOI: 10.1043/0003-3219(1981)051<0115:PITCAO>2.0. $\mathrm{CO} ; 2$.

10. Ricketts RM. Cephalometric Analysis and Synthesis. Angle Orthod. 1961;31(3):141-56. DOI: 10.1043/0003-3219(1961)031<0141:CAAS>2.0.CO;2.

11. Howe RP, McNamara JA Jr., O'Connor KA. An examination of dental crowding and its relationship to tooth size and arch dimension. Am J Orthod. 1983;83(5):36373. DOI: $10.1016 / 0002-9416(83) 90320-2$.

12. McNamara JA Jr. Long-term adaptations to changes in the transverse dimension in children and adolescents: an overview. Am J Orthod Dentofacial Orthop. 2006;129(4 Suppl):S71-S74. DOI: 10.1016/j.ajodo.2005.09.020.

13. Lemieux G, Carey JP, Flores-Mir C, Secanell M, Hart A, Dietrich $\mathrm{N}$, et al. Three-dimensional cephalometric superimposition of the nasomaxillary complex. Am J Orthod Dentofacial Orthop. 2014;146(6):758-64. DOI: 10.1016/j.ajodo.2014.08.014.

14. Sawchuk D, Currie K, Lagravere M, Martin J, Flores-Mir C. Diagnostic methods for assessing maxillary skeletal and dental transverse deficiencies: A systematic review. The Korean J Orthod. 2016;46(5):331. DOI: 10.4041/ kjod.2016.46.5.331.

15. Varghese S, Kailasam V, Padmanabhan S, Vikraman B, Chithranjan A. Evaluation of the accuracy of linear measurements on spiral computed tomography-derived-three-dimensional images and its comparison with digital cephalometric radiography. Dentomaxillofac Radiol. 2010;39(4):216-23. DOI: $10.1259 / \mathrm{dmfr} / 30048377$.

16. Lagravere MO, Major PW, Flores-Mir C. Long-term skeletal changes with rapid maxillary expansion: a systematic review. Angle Orthod. 2005;75(6):1046-52. DOI: 
10.1043/0003-3219(2005)75[1046:LSCWRM]2.0. $\mathrm{CO} ; 2$.

17. Brunetto DP, Sant'Anna EF, Machado AW, Moon W. Non-surgical treatment of transverse deficiency in adults using Microimplant-assisted Rapid Palatal Expansion (MARPE). Dental Press J Orthod. 2017;22(1):110-25. DOI: $10.1590 / 2177-6709.22 .1 .110-125 . s a r$.

18. Lee KJ, Park YC, Park JY, Hwang WS. Miniscrew-assisted nonsurgical palatal expansion before orthognathic surgery for a patient with severe mandibular prognathism. Am J Orthod Dentofacial Orthop. 2010;137(6):830-9. DOI: 10.1016/j.ajodo.2007.10.065.

19. Mutinelli S, Cozzani M. Rapid maxillary expansion in early-mixed dentition: effectiveness of increasing arch dimension with anchorage on deciduos teeth. Eur J Paediatr Dent [Internet]. 2015 [citado el 5 de septiembre del 2020]; 16(2):115-122. Disponible en: https://pubmed. ncbi.nlm.nih.gov/26147817/.

20. Rosa M, Lucchi P, Manti G, Caprioglio A. Rapid Palatal Expansion in the absence of posterior cross-bite to intercept maxillary incisor crowding in the mixed dentition: a CBCT evaluation of spontaneous changes of untouched permanent molars. Eur J Paediatr Dent [Internet]. 2016 [citado el 5 de septiembre del 2020]; 17(4):286294. Disponible en: https://pubmed.ncbi.nlm.nih. gov/28045316/.

21. Spillane LM, McNamara JA Jr. Maxillary adaptation to expansion in the mixed dentition. Semin Orthod. 1995;1(3):176-87. DOI: 10.1016/s10738746(95)80021-2.

22. Memikoglu TU, Işeri H. Effects of a bonded rapid maxillary expansion appliance during orthodontic treatment. Angle Orthod. 1999;69(3):251-6. DOI: 10.1043/0003-3219(1999)069<0251:EOABRM>2.3. $\mathrm{CO} ; 2$.

23. Geran RG, McNamara JA Jr, Baccetti T, Franchi L, Shapiro LM. A prospective long-term study on the effects of rapid maxillary expansion in the early mixed dentition. Am J Orthod Dentofacial Orthop. 2006;129(5):631-40. DOI: 10.1016/j.ajodo.2005.01.020.

24. Bishara SE, Staley RN. Maxillary expansion: clinical implications. Am J Orthod Dentofacial Orthop. 1987;91(1):3-14. DOI: 10.1016/0889-5406(87)902022.

25. Gryson JA. Changes in mandibular interdental distance concurrent with rapid maxillary expansion. Angle Orthod. 1977;47(3):186-92. DOI: 10.1043/0003-3219(1977)047<0186:CIMIDC>2.0. $\mathrm{CO} ; 2$.

26. Tollaro I, Baccetti T, Franchi L, Tanasescu CD. Role of posterior transverse interarch discrepancy in Class II, Division 1 malocclusion during the mixed dentition phase. Am J Orthod Dentofacial Orthop. 1996;110(4):417-22. DOI: $10.1016 / \mathrm{s} 0889-5406(96) 70045-8$.

27. Gianelly AA. Rapid palatal expansion in the absense of crossbites: added value? Am J Orthod Dentofacial Orthop. 2003;124(4):362-5. DOI:10.1016/s08895406(03)00568-7.

28. Guest SS, McNamara JA Jr, Baccetti T, Franchi L. Improving Class II malocclusion as a side-effect of rapid maxillary expansion: A prospective clinical study. Am J Orthod Dentofacial Orthop. 2010;138(5):582-91. DOI: 10.1016/j.ajodo.2008.12.026.

29. Chung $\mathrm{CH}$, Font B. Skeletal and dental changes in the sagittal, vertical, and transverse dimensions after rapid palatal expansion. Am J Orthod Dentofacial Orthop. 2004;126(5):569-75. DOI: 10.1016/j.ajodo.2003.10.035.

30. Reichenbach E, Taatz K. Kieferorthopaedische Klinik und Therapie. Heft 7. Leipzig, Germany: JA Barth Verl; 1971.

31. Haas AJ. Rapid expansion of the maxillary dental arch nasal cavity by opening the midpalatal suture. Angle Orthod. 1961;31(2):73-90. DOI: 10.1043/0003-3219(1961)031<0073:REOTMD>2.0. $\mathrm{CO} ; 2$.

32. Cameron CG, Franchi L, Baccetti T, McNamara JA Jr. Long-term effects of rapid maxillary expansions: a posteroanterior cephalometric evaluation. Am J Orthod Dentofacial Orthop. 2002;121(2):129-35. DOI: $10.1067 /$ $\bmod .2002 .120685$.

33. Lione R, Brunelli V, Franchi L, Pavoni C, Quiroga B, Cozza P. Mandibular response after rapid maxillary expansion in class II growing patients: a pilot randomized controlled trial. Prog Orthod. 2017;18(1):36. DOI: 10.1186/s40510-017-0189-6.

34. Baratieri C, Alves M Jr, Sant'anna EF, Nojima Mda C, Nojima LI. 3D mandibular positioning after rapid maxillary expansion in Class II malocclusion. Braz Dent J. 2011;22(5):428-34. DOI: 10.1590/s010364402011000500014 .

35. Ugolini A, Doldo T, Ghislanzoni LT, Mapelli A, Giorgetti R, Sforza C. Rapid palatal expansion effects on mandibular transverse dimensions in unilateral posterior crossbite patients: a three-dimensional digital imaging study. Prog Orthod. 2016;17:1. DOI: 10.1186/s40510015-0114-9.

36. Di Ventura A, Lanteri V, Gaffuri F, Farronato G, Beretta M, Cossellu G, et al. Three-dimensional evaluation of rapid maxillary expansion anchored to primary molars: direct effects on maxillary arch and spontaneous mandibular response. Eur J Paediatr Dent. 2019;20(1):38-42. DOI: 10.23804/ejpd.2019.20.01.08.

37. Melgaço CA, Columbano Neto J, Jurach EM, Nojima Mda C, Sant'Anna EF, Nojima LI. Rapid maxillary expansion effects: an alternative assessment method by means of cone-beam tomography. Dental Press J Orthod. 2014;19(5):88-96. DOI: 10.1590/21769451.19.5.088-096.oar.

38. McNamara JA Jr, Brudon WL. Orthodontics and Dentofacial Orthopedics. Ann Arbor, Mich: Needham Press; 1995.

39. O'Grady PW, McNamara JA Jr, Baccetti T, Franchi L. A long-term evaluation of the mandibular Schwarz appliance and the acrylic splint expander in early mixed dentition patients. Am J Orthod Dentofacial Orthop. 2006;130(2):202-13. DOI: 10.1016/j.ajodo.2004.12.023.

40. Tai K, Park JH. Dental and skeletal changes in the upper and lower jaws after treatment with Schwarz appliances 
using cone-beam computed tomography. J Clin Pediatr Dent. 2010;35(1):111-20. DOI: 10.17796/jcpd.35.1.b$21 \mathrm{t} 44 \mathrm{rj3} 3027603$.

41. Tai K, Park JH, Mishima K, Shin JW. 3-Dimensional cone-beam computed tomography analysis of transverse changes with Schwarz appliances on both jaws. Angle Orthod. 2011;81(4):670-7. DOI: 10.2319/110910655.1.

42. Guerrero CA. Expansion mandibular quirurgica. Rev Venez Ortod [Internet]. 1990 [citado el 22 de septiembre del 2020]; 7(1/2):48-50. Disponible en: https://pesquisa.bvsalud.org/portal/resource/pt/lil-127161.

43. Guerrero CA, Bell WH, Contasti GI, Rodriguez AM. Mandibular widening by intraoral distraction osteogenesis. Br J Oral Maxillofac Surg. 1997;35(6):383-92. DOI: 10.1016/s0266-4356(97)90712-9.
44. Del Santo M Jr, English JD, Wolford LM, Gandini LG Jr. Midsymphyseal distraction osteogenesis for correcting transverse mandibular discrepancies. Am J Orthod Dentofacial Orthop. 2002;121(6):629-38. DOI: 10.1067/ $\bmod .2002 .122240$.

45. Starch-Jensen T, Kjellerup AD, Blæhr TL. Mandibular midline distraction osteogenesis with a Bone-borne, Tooth-borne or Hybrid Distraction Appliance: a Systematic Review. J Oral Maxillofac Res. 2018;9(3):e1. DOI: $10.5037 /$ jomr.2018.9301. 